Ann. Génét. Sél. anim., I973, 5 (4), 507-518.

REVUE BIBLIOGRAPHIQUE

\title{
LES ALLOANTIGÈNES \\ SUR LES SPERMATOZOÏDES DES MAMMIFÈRES LEUR CONTRÔLE PAR LE GÉNOME HAPLOÏDE
}

\author{
C. PRAKASH ( $\left.{ }^{1}\right)$ \\ Laboratoive de Génétique biochimique \\ Centre national de Recherches zootechniques, I. N. R. A., \\ 78350 Jouy en Josas
}

RÉSUMÉ

Cette revue bibliographique fait le point des travaux qui ont tenté de révéler la présence d'alloantigènes cellulaires sur les spermatozoïdes des mammifères. Les investigations ont porté sur les systèmes humains $\mathrm{ABO}, \mathrm{MNS}, \mathrm{Rh}, \mathrm{P}(\mathrm{Tja}), \mathrm{Xg} a$ et HLA, sur les systèmes bovins $\mathrm{A}, \mathrm{B}, \mathrm{C}$, $\mathrm{F}, \mathrm{Z}, \mathrm{M}$ et $\mathrm{J}$, sur les systèmes $\mathrm{H}-2$ et $\mathrm{H}-\mathrm{Y}$ de la Souris et sur le système $\mathrm{Hg}$ du Lapin.

Les résultats de ces travaux sont très discordants, ce qui semble pouvoir s'expliquer par l'inadéquation des techniques qui ont été utilisées dans bien des cas. Dans l'ensemble, à la lumière de nos conceptions immunologiques actuelles, les études concluant à la présence d'alloantigènes sur les spermatozoïdes semblent plus convaincantes que celles qui concluent à leur absence.

Il reste aussi à démontrer de manière définitive que ces alloantigènes sont synthétisés par les spermatozoïdes, donc génétiquement déterminés par le génome haploïde dont ces derniers sont vecteurs. Les travaux les plus récents paraissent confirmer cette hypothèse, qui semble par ailleurs compatible avec nos connaissances actuelles de la biologie des spermatozoïdes.

Le fractionnement de sous-populations de spermatozoïdes à l'aide de techniques immunologiques pourrait donc être envisagé dans l'avenir.

\section{INTRODUCTION}

Les seuls liens entre les générations animales sont les gamètes qui assurent la transmission de l'information héréditaire d'une génération à l'autre.

Il n'a pas été encore définitivement prouvé que les spermatozoïdes des mammifères possédaient, comme toutes les autres cellules, des déterminants antigéniques leur conférant une marque

(') Adresse actuelle : Division of Genetics, National Dairy Research Institute, Karnal, Haryana, India.

Annales de Génétique animale. - 1973. 
individuelle. Les résultats obtenus jusqu'ici dans ce domaine sont nettement contradictoires. En fait, les études nécessaires impliquent une approche interdisciplinaire, touchant aussi bien à la génétique qu'à la physiologie et à l'immunologie de la reproduction. La relative complexité du problème explique pourquoi on a souvent mis en cuvre des techniques défectueuses et abouti à des conclusions hâtives, insuffisamment étayées par une expérimentation rigoureuse.

C'est semble-t-il à Metchnikoff, de l'Institut Pasteur de Paris, qu'il faut attribuer la première tentative d'étudier l'antigénicité des spermatozoïdes des mammifères (I 899, I900). TyLER (I96I) a passé en revue la plupart des premières expériences, et un certain nombre de mises au point (Metz, i967 ; Beatty, I970 ; Papivanov et Vulchanov, i969; Cohen, i971 ; Shulman, I97I $a$, I97I $b$ et 1972) ont été publiées depuis.

EDWARds et al. (1964) mettent bien en évidence l'intérêt que de nombreux auteurs ont porté à l'immunologie des spermatozoïdes humains avec l'espoir, notamment, de déceler sur ces derniers, des antigènes de groupes sanguins ou d'autres alloantigènes. Cet intérêt s'explique par les possibilités d'applications intéressantes que l'on a cru entrevoir, et qui peuvent se résumer brièvement ainsi :

Supposons que l'action d'un gène se traduise, après la ségrégation gamétique, donc à l'état haploïde, par un dimorphisme antigénique dans une population de spermatozoïdes individuelle. En recourant à des techniques immunologiques, on pourrait soit empêcher l'une des deux souspopulations de spermatozoïdes de participer à la fécondation in vivo ou in vitro, soit séparer les deux sous-populations in vitro avant la fécondation. Sous certaines conditions, ces techniques pourraient permettre de contrôler le sexe, de prévenir l'incompatibilité Rhésus ou encore d'éviter la transmission de gènes léthaux. Par ailleurs, on a pu considérer que l'antigénicité des spermatozoïdes pouvait être la cause de certaines stérilités mal expliquées ou d'aptitudes à la reproduction réduites. Une meilleure connaissance de cette antigénicité pourrait contribuer à résoudre ce type de problèmes.

Les travaux effectués jusqu'ici ont porté sur des antigènes des systèmes $\mathrm{ABO}, \mathrm{MNS}, \mathrm{Rh}$, $\mathrm{P}(\mathrm{Tja})$ et $\mathrm{Xg}^{a}$, HL-A humains, sur des antigènes appartenant aux systèmes de groupes sanguins bovins A, B, C, F, Z, M et J, sur des antigènes des systèmes H-2 et H-Y de la Souris et sur des antigènes du système $\mathrm{Hg}$ du Lapin.

On doit noter que les antigènes $A$ et $B$ du système humain $A B O$ (RACE et SANGER, 1962) et l'antigène J du système bovin J (YCAS, I949; Stormont, I949; Stone et IRwin, 1954) existent dans des sécrétions telles que la salive, le plasma séminal, le plasma sanguin, etc. On ne peut donc pas exclure l'hypothèse que les antigènes de ce type présents sur les spermatozoïdes proviennent d'une adsorption non spécifique à partir du plasma séminal. Il n'est pas possible en effet de distinguer clairement à l'aide des techniques immunologiques actuelles ceux des antigènes qui font effectivement partie de la membrane des spermatozoïdes de ceux qui proviennent du plasma séminal. A noter que des auteurs comme Morgenstern (r968) admettent que l'adsorption des antigènes $\mathrm{A}$ et $\mathrm{B}$ des donneurs sécréteurs est irrévocable.

\section{A. - TRAVAUX CONCLUANT A LA PRÉSENCE D'ALLOANTIGÈnES SUR LES SPERMATOZOÏDES}

Dès I926, YAMAKAmI a démontré la présence des substances de groupes sanguins du système ABO humain dans la semence entière et dans le plasma séminal en procédant par inhibition spécifique des isohémagglutinines naturelles. La même année, LANDSTEINER et Levine (i926) ont développé une technique d'absorption similaire ; utilisant un immunsérum de lapin et des spermatozoïdes (lavés une fois) de seize donneurs qui appartenaient à divers groupes du système ABO, ces auteurs ont conclu que les spermatozoïdes humains possédaient des substances identiques ou similaires aux facteurs iso-agglutinables A et B des érythrocytes humains. LANDSTEINER et 
LEvine estimaient avoir éliminé, des préparations qu'ils utilisaient pour les absorptions, la plupart des cellules prostatiques lourdes et des particules en suspension; il nous paraît toutefois douteux, à la lecture du protocole expérimental, qu'ils aient réussi à obtenir des préparations de spermatozoïdes suffisamment propres pour que leurs conclusions puissent être acceptées sans réserves. La distinction entre donneurs sécréteurs et non-sécréteurs a été découverte plus tard (Lehrs, I930; Putkonen, I930; Friedenreich et Hartmann, I938; Hartmann, i94i). Levine et Celano (I96I), en utilisant des techniques similaires, ont confirmé les résultats de LANDSTEINER et LEVINE (I926), en tenant compte du caractère sécréteur ou non-sécréteur des donneurs de spermatozoïdes.

La présence des antigènes des groupes sanguins sur les spermatozoïdes humains a été montrée également par Krainskaya-Ignatova (1929); Maligina (1949); Papivanov et Ermenkova (r960), qui, tous, utilisaient des techniques similaires.

Docron et al. (1952), en utilisant des réactifs monospécifiques iso-immuns contre les antigènes des globules rouges bovins ont démontré par une technique d'inhibition d'hémolyse que la plupart des facteurs antigéniques de groupes sanguins connus à l'époque étaient présents sur les spermatozoïdes. Cependant, les résultats divergents observés avec certains réactifs chez des sujets différents jettent un doute sur ces résultats : c'est ainsi que les facteurs $\mathrm{Y}$ (système B) et $\mathrm{F}$ (système F) présents l'un et l'autre sur les hématies de deux des trois taureaux utilisés n'ont été décelés sur les spermatozoïdes que de l'un d'entre eux. Les auteurs de ce travail ont interprété les résultats des tests d'absorption d'anticorps comme une confirmation des tests d'inhibition d'hémolyse. Ils rapportent par ailleurs la production d'anticorps spécifiques de certains antigènes par hétéroimmunisation à l'aide des spermatozoïdes d'un taureau. Autant que l'on puisse en juger, il n'est pas certain que ce travail ait été mené avec une rigueur suffisante. De plus, le nombre d'échantillons est faible et les cellules contaminantes ainsi que les débris cellulaires n'ont pas été éliminés, ce qui peut conduire à remettre en cause toutes les conclusions.

Gullbring (1957), utilisant la technique d'agglutination mixte de cellules due à Coombs et al. (1956), rapporte la mise en évidence des antigènes $\mathrm{A}$ et $\mathrm{B}$ du système $\mathrm{ABO}$ et de l'antigène $\mathrm{D}$ du système Rh sur les spermatozoïdes humains, et décrit la séparation, à partir du sperme de donneurs de groupe $\mathrm{AB}$, de deux sous-populations de spermatozoïdes portant respectivement l'antigène $A$ et l'antigène $B$. La suspension indicatrice de GullBRING consistait, pour le système $\mathrm{ABO}$, en des érythrocytes non traités et pour le système $\mathrm{Rh}$, en des érythrocytes traités avec un enzyme (trypsine ou papaïne). Bien qu'il n'ait pas été établi si les donneurs étaient ou non sécréteurs, les spermatozoïdes ont été lavés avant et après leur incubation en présence des anticorps. Dans ce travail de Gullbring, seuls les résultats caractéristiques de chaque groupe sont représentés et l'absence de plusieurs précisions relatives au protocole expérimental ne permet pas une évaluation critique des résultats.

Papivanov et Vulchanov (I962) ont présenté des résultats tendant à confirmer ceux de Gullbring. Utilisant le test direct d'agglutination du sperme avec l'anti-A hétéro-immun monospécifique, produit en immunisant des lapins avec des spermatozoïdes d'un donneur de groupe sanguin A, ces auteurs ont conclu que les spermatozoïdes des donneurs AB représentent un mélange de deux populations, possédant l'une l'antigène $\mathrm{A}$, l'autre l'antigène $\mathrm{B}$. Plus tard, Vulchanov et Papivanov (1972), en se basant sur les mêmes résultats, ont soutenu plus nettement encore la thèse du dimorphisme des populations de spermatozoïdes. Cependant, Spooner (1972) a émis des doutes sur la spécificité de l'anti-A hétéro-immun employé dans ces expériences.

ShaHanI et Southam (1962) ont directement utilisé la technique d'immunofluorescence sur des frottis de spermatozoïdes fixés par l'éther, provenant de seize donneurs de groupes sanguins $\mathrm{A}, \mathrm{B}, \mathrm{AB}$ et $\mathrm{O}$ et comptant parmi eux des sécréteurs et des non-sécréteurs. Ils ont montré, de la sorte, aussi bien la présence d'antigènes spécifiques des groupes sanguins que la ségrégation de ces antigènes sur les spermatozoïdes humains, et n'ont pas observé de différence qualitative de fluorescence entre les sécréteurs et les non-sécréteurs. Les détails du protocole expérimental suivi par ces auteurs paraissent convaincants. L'affinité spécifique des anticorps marqués apparaît dans le résumé des résultats de Shahani et Southam (I962) présenté ci-après : 
Anticorps

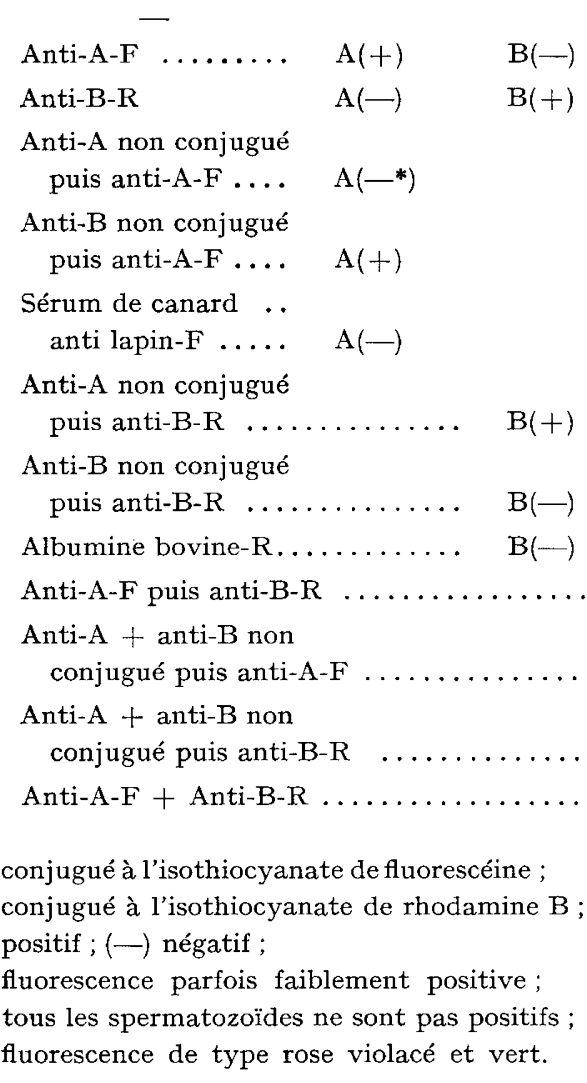

Le dimorphisme de la population des spermatozoïdes provenant des donneurs $\mathrm{AB}$ a été également établi d'une manière rigoureuse. Il est cependant curieux que ces auteurs aient pratiqué une durée d'incubation inhabituellement longue pour des frottis de spermatozoïdes fixés avec des sérums conjugués ( $\mathrm{r}$ heure d'incubation à $37^{\circ} \mathrm{C}$, puis pendant une nuit à $4^{\circ} \mathrm{C}$ ). Autant que l'on sache, la technique d'immunofluorescence ne requiert pas, pour être efficace, une si longue durée d'incubation. Comme les antisérums étaient utilisés non dilués, on peut supposer la présence d'une petite quantité de ces antigènes sur les spermatozoïdes. Il est dommage que ShaHani et Southam n'aient pas publié des résultats quantitatifs.

ACKerman (1969) s'est attaqué au problème par une approche indirecte en mettant en œuvre la technique de microcataphorèse cellulaire qui permet de quantifier des différences d'absorption par les protéines, les polysaccharides ou les lipides des surfaces cellulaires. Dans certaines conditions, cette technique permet de distinguer un dimorphisme antigénique dans une population de cellules. En utilisant des contrôles appropriés et des techniques statistiques pour l'analyse de ses données, Ackerman a montré que les spermatozoïdes humains possèdent des antigènes correspondant au système $\mathrm{ABO}$, que le donneur soit ou non sécréteur.

PADMA (1969) a étudié, chez les bovins, la relation entre les antigènes des groupes sanguins et les antigènes homologues des spermatozoïdes, en utilisant des agglutinines naturelles non définies : l'interprétation de ses résultats est, comme le sont les conclusions de Docton et al. (1952), en faveur de l'existence d'au moins quelques facteurs antigéniques de groupes sanguins sur les spermatozoïdes. En réalité, la technique modifiée d'agglutination mixte de cellules, utilisée par 
Padma, n'était pas appropriée. En effet, les spermatozoïdes n'ayant pas été lavés avant l'addition de la suspension de cellules indicatrices, le milieu de suspension contenait probablement une quantité assez importante d'anticorps pouvant avoir une affinité suffisante pour réagir avec les cellules indicatrices seules. L'antigène I (non défini) des érythrocytes a été trouvé sur les spermatozoïdes de cinq des huit taureaux testés, alors que l'antigène II (non défini) des érythrocytes était absent sur les spermatozoïdes des huit taureaux testés.

Vojtiskova et al. (1969) ont démontré, à l'aide de plusieurs techniques immunologiques, la présence d'antigènes d'histocompatibilité sur les spermatozoïdes de Souris. Les nombres de cellules de la rate, de cellules du sang et de spermatozoïdes des donneurs positifs nécessaires pour absorber une certaine quantité d'antisérum correspondant, de titre élevé, étaient dans le rapport I, 2 et 4 . Dans une étude semi quantitative, ces auteurs ont montré qu'à peu près 400 millions de spermatozoïdes étaient nécessaires pour épuiser complètement $0,2 \mathrm{ml}$ d'anti-sérum de titre élevé. Ils estiment à ro p. Ioo le nombre maximum de cellules contaminantes (autres que des spermatozoïdes) présentes dans leurs préparations. Ils estiment également que, jusqu'à un seuil de 5o millions, les cellules de la rate ou du sang n'affectent pas significativement le titre de l'antisérum dans leurs expériences. Ceci constitue une preuve indirecte de la non-intervention des cellules autres que les spermatozoïdes dans les absorptions réalisées avec leurs préparations. Il semble que Vojriskova et al. aient démontré de manière convaincante la présence sur les spermatozoïdes des souris, des facteurs antigéniques commandés par les allèles $\mathrm{H}-2^{a}$ et $\mathrm{H}-2^{d}$, du système $\mathrm{H}-2$. En utilisant par la suite la méthode indirecte d'immunofluorescence, ces auteurs ont apporté une autre preuve de la présence des facteurs antigéniques des allèles $\mathrm{H}-2^{a}, \mathrm{H}_{-2}{ }^{b}, \mathrm{H}-2^{d}, \mathrm{H}-2^{k} \mathrm{du}$ système $\mathrm{H}-2$, quoique certains antisérums donnent une fluorescence faiblement positive dans leur système. Les résultats des tests de présensibilisation avant les transplantations de peaux sont interprétés dans leur travail en faveur de la présence sur les spermatozoïdes des souris des produits antigéniques de l'allèle $\mathrm{H}-3^{a}$ et/ou $\mathrm{H}-\mathrm{r} 3^{a}, \mathrm{H}-2^{a}$, et d'un antigène spécifique du sexe masculin. De plus, en utilisant seulement des spermatozoïdes épididymaires, ces auteurs ont éliminé le risque d'adsorption de ces antigènes à partir du plasma séminal. De toutes façons, les antigènes des systèmes d'histocompatibilité n'ont été détectés, sous forme soluble, dans aucune des espèces étudiées jusqu'à maintenant.

En utilisant une micro- et une macro-technique de cytotoxicité, Fellous et Dausser (I970) ont démontré la présence d'antigènes HL-A : $\left(A_{1}, A_{2}, A_{5}, A_{7}, A_{8}\right.$ et $\left.A_{9}\right)$ et $\mathrm{Da}\left(\mathrm{Da}_{4}\right.$ et $\left.\mathrm{Da}_{14}\right)$ sur les spermatozoïdes humains de neuf donneurs dont les lymphocytes possédaient ces antigènes. De plus, ils ont confirmé ce résultat en utilisant un antisérum anti HL- $\mathrm{A}_{2}$ absorbé par les plaquettes de trois donneurs. Le sérum absorbé ne réagissait ni avec les spermatozoïdes des trois donneurs correspondants, ni avec leurs lymphocytes, bien que l'éluat des plaquettes des donneurs fût actif avec les spermatozoïdes et les lymphocytes d'un donneur testé. Parmi les neuf donneurs, quatre étaient hétérozygotes au premier locus (les gènes contrôlant HL- $\mathrm{A}_{1}, \mathrm{~A}_{2}, \mathrm{~A}_{3}, \mathrm{~A}_{9}, \mathrm{Da}_{15}, \mathrm{Da}_{17}$ s'excluent mutuellement et sont donc allèles) et trois autres étaient hétérozygotes pour le deuxième locus (les gènes sur ce locus commandent les antigènes $\mathrm{HL}_{\mathrm{f}} \mathrm{A}_{5}, \mathrm{~A}_{2}, \mathrm{~A}_{8}$ et $\mathrm{Da}_{4}$ ). Seuls, deux donneurs étaient homozygotes pour le premier locus (antigène $\mathrm{HL}-\mathrm{A}_{2}$ ). Douze mélanges d'antisérum faits dans le but de tester soit les homozygotes, soit les hétérozygotes en configuration trans ont donné 67 p. Ioo de spermatoxicité, alors que cinq autres mélanges destinés à tester les antigènes contrôlés par les gènes en configuration cis ont donné 47 p. Ioo de spermatotoxicité. Le degré de spermatoxicité dans les témoins était de Io-I 5 p. Ioo. La différence de spermatotoxicité entre donneurs homozygotes et hétérozygotes n'a été attribuée ni à l'effet de la dose des anticorps, ni à leur action synergétique. Les résultats quantitatifs ont été interprétés comme suggérant une expression haploïde du locus HL-A sur les spermatozoïdes humains.

Le travail de Fellous (I969) utilisant les mêmes techniques avec les spermatozoïdes de Souris, étend ces résultats à la mise en évidence d'une expression haploïde pour le système $\mathrm{H}-2$. Il est vraisemblable que le perfectionnement des techniques de préparation des spermatozoïdes et la recherche des conditions optimales pour la technique de microcytotoxicité réduiront le degré de spermatotoxicité dans les témoins. Ces ajustements permettront ainsi d'augmenter le degré 
de spermatotoxicité de l'antisérum vis-à-vis des spermatozoïdes de donneurs homozygotes ou du mélange d'anticorps destiné à tester la présence d'antigènes en configuration trans. L'expression des valeurs de cytotoxicité obtenues en présence d'anticorps par rapport à celles des témoins sous la forme de l'index de spermatotoxicité développé par HammerLynck et RUMKE (I968) pourrait améliorer la comparaison, donc faciliter l'interprétation des données quantitatives.

GoldBerg et al. (1970) en utilisant la même technique de cytotoxicité, ont détecté la présence de produits antigéniques de $\mathrm{H}_{-2} \mathbf{2}^{b}, \mathrm{H}-2^{k}, \mathrm{H}-2^{b a}$, et $\mathrm{H}-2^{a}$ (allèles du système $\mathrm{H}-2$ ) sur les spermatozoïdes prélevés dans l'épididyme ou dans le canal déférent de la souris. Les spermatozoïdes de l'épididyme ont donné, en l'absence de lavage, une réaction plus forte que ceux du canal déférent. Il est probable que le plasma séminal absorbé sur les spermatozoïdes intervient dans le mécanisme de la réaction (par exemple par son effet anticomplémentaire).

En utilisant la même technique, GoLDBERG et al. (I97I) ont également démontré la présence de l'antigène H-Y (lié au sexe) sur les spermatozoïdes des souris. La spécificité de cette technique est encore mise en évidence, dans leur travail, par l'absence sur les spermatozoïdes des antigènes de la différenciation du système lymphoïde (TL-O, LY-A et LY-B). GoLDBERG (1972) a confirmé ces résultats préliminaires dans un travail plus complet et détaillé.

\section{B. - TRAVAUX CONCLUANT A L'ABSENCE D'ALLOANTIGÈNES SUR LES SPERMATOZOÏDES}

Un certain nombre d'auteurs ont contesté la présence d'alloantigènes sur les spermatozoïdes des mammifères.

SJÖVALL (1948-1950) n'a pas trouvé de différence entre les homozygotes et les hétérozygotes pour le système $\mathrm{ABO}$ en se servant des techniques d'agglutination du sperme ou de spermiolyse chez onze donneurs dont un seul était un A non sécréteur.

Holborow et al. (1960) en appliquant la méthode d'immunofluorescence indirecte sur des spermatozoïdes testiculaires de quatre adultes de groupe sanguin A et sur des coupes de deux testicules d'enfants n'ont pas trouvé de fluorescence spécifique.

Levine et Celano (196I) n'ont pu démontrer la présence sur les spermatozoïdes de l'antigène $\mathrm{D}$ du système $\mathrm{Rh}$ par la technique d'absorption et d'élution. Rappelons que ces auteurs avaient, par contre, détecté par la même technique, les antigènes du système ABO.

Quinlivan et Masouredis (I962) en utilisant l'anticorps anti-Rho (ou anti-D) marqué avec ${ }^{131} \mathrm{I}$, n'ont pas trouvé de différence quantitative dans la fixation d'anticorps marqués sur des spermatozoïdes de dix donneurs $\mathrm{D}$ positifs et de cinq donneurs D négatifs. Les mêmes auteurs ont aussi recherché l'antigène $\mathrm{D}$ sur les spermatozoïdes de leurs donneurs par la technique d'agglutination de cellules mixtes et ont obtenu des résultats positifs chez deux des huit donneurs $\mathrm{D}$ positifs mais aussi chez quatre des six donneurs $\mathrm{D}$ négatifs. Toutefois, il est étonnant que ces auteurs n'aient pas détecté, dans leurs expériences, de différence quantitative entre la suspension indicatrice d'érythrocytes $\mathrm{D}$ positifs et $\mathrm{D}$ négatifs. Cette anomalie conduit à douter de la spécificité des anti-sérums anti-D qu'ils ont utilisés.

BARTH et Russell (1964), après avoir fixé par l'acétone froide des spermatozoïdes de Souris, ne sont pas parvenus à mettre en évidence, sur ces spermatozoïdes, par la technique d'immunofluorescence directe et indirecte, les produits antigéniques de l'allèle $\mathrm{H}_{-2}{ }^{a}$.

Mais dans une expérience similaire, Vojtiskova, Polackova et Pokorna (r969) ont observé une faible immunofluorescence qu'ils ont interprétée comme preuve de la présence de l'antigène d'histocompatibilité sur les spermatozoïdes. La même conclusion a été tirée des résultats obtenus par absorption et présensibilisation des receveurs de greffes de peau. Ces résultats contradictoires 
peuvent s'expliquer par une mauvaise interprétation des réactions faibles mais spécifiques observées avec la technique d'immunofluorescence.

Schmid et al. (1964) ont testé, par la technique d'inhibition de l'hémolyse, les spermatozoïdes lavés de $\mathrm{r} 22$ taureaux de trois races différentes avec les réactifs spécifiques des groupes sanguins. Un seul des $4^{\circ}$ réactifs examinés a donné des résultats positifs (anti-J). L'antigène $\mathrm{J}$ était également détectable dans le plasma séminal. Nous pensons que la sensibilité insuffisante de la technique classique d'inhibition de l'hémolyse ne permet pas de détecter la petite quantité d'alloantigènes présente sur les spermatozoïdes. Les mêmes auteurs ont immunisé des lapins avec les spermatozoïdes pour produire des anticorps spécifiques des antigènes érythrocytaires. Leurs résultats négatifs peuvent aussi s'expliquer par la présence d'une trop petite quantité d'alloantigènes sur les spermatozoïdes.

EDWARDs et al. (1964) ont étudié la présence de plusieurs alloantigènes, sur les spermatozoïdes humains. En utilisant la technique d'agglutination mixte, ces auteurs ont conclu à l'absence des antigènes $\mathrm{A}$ et $\mathrm{B}$ sur les spermatozoïdes des donneurs non-sécréteurs correspondants. Pourtant, quoique cet aspect de leur travail soit souvent passé sous silence, EDwards et al. montrent, par la même technique, la présence des antigènes $M$ et $N$ de système MNS et de l'antigène $\mathrm{Tja}$ du système actuellement dénommé $\mathrm{P}$; on ne peut donc pas conclure, sur la base de ce travail, à l'absence d'alloantigènes sur les spermatozoïdes.

Les expériences de QuinLIVAN et Masouredrs (I962) nous invitent à considérer les résultats d'EDwards et al. avec prudence. En effet, compte tenu des dimensions des érythrocytes $(7,5 \mu$ selon Ponder, 1948), et de la tête des spermatozoïdes humains $(4,2 \mu$ et $9,2 \mu$ selon SchulTz-LARSEN, 1958) et connaissant la possibilité d'agglutination non spécifique de ces spermatozoïdes (COHEN, I97I), il semble évident que ces derniers ne présentent pas la même sensibilité, ni la même spécificité à la technique d'agglutination mixte que les autres cellules telles que les fibroblastes. Il est également probable que de petites quantités d'alloantigènes de spermatozoïdes humains ne sont pas détectables par cette technique par suite d'empêchement stérique. Cependant, EDwards et al. indiquent que la technique d'agglutination mixte ne permet pas de détecter les antigènes situés en profondeur de la membrane des spermatozoïdes. Ils n'ont pas réussi par ailleurs, à établir la présence d'antigène $\mathrm{Xg}^{a}$ sur les spermatozoïdes des deux donneurs testés par la technique d'agglutination antiglobulinique de cellules mixtes mais ces résultats ne devraient pas être considérés comme concluants car les deux donneurs n'ont été testés qu'une fois avec l'antisérum anti- $\mathrm{Xg}^{a}$ faible utilisé avec le sérum anti-globuline humain servant aux groupes du système $\mathrm{Rh}$. Ce sérum antiglobuline était certainement riche en anti-IgG. Il est très probable que son efficacité eût été moins grande si l'activité de l'anticorps anti-Xg ${ }^{a}$ résidait ailleurs que dans la fraction IgG. Prakash (r973 b) indique que les anticorps du type $\operatorname{IgM}$ se lient plutôt avec les récepteurs de la membrane du spermatozoïde humain qu'avec les anticorps de type IgG de la même spécificité.

BOEtTCHer (1965) a démontré in vitro que les spermatozoïdes adsorbaient du plasma séminal contenant des antigènes du système $\mathrm{ABO}$, mais ses expériences n'excluent d'aucune façon la présence éventuelle de petites quantités de ces antigènes sur les spermatozoïdes non sécréteurs.

Utilisant une technique d'agglutination antiglobulinique de cellules mixtes, PARIsH $e t$ al. (1967) ont obtenu une réaction faiblement positive dans certains cas avec deux donneurs non sécréteurs $\mathrm{A}$, et une réaction négative avec les deux autres donneurs non sécréteurs $\mathrm{A}$. On peut estimer cependant que ces techniques d'agglutination de cellules mixtes manquent de spécificité dans le cas où des érythrocytes sont employés comme suspension indicatrice pour la détection d'antigènes sur la membrane de spermatozoïdes humains.

MoRgenstern (1968) n'a pas non plus réussi à détecter d'antigènes du système $\mathrm{ABO}$ sur les spermatozoïdes humains. Sa technique d'agglutination mixte présente les mêmes désavantages que celles mentionnées précédemment et sa technique d'inhibition qualitative n'a pas la précision requise pour la détection de petites quantités d'antigènes.

$\mathrm{Au}$ cours de tentatives de détection d'antigènes H-2 chez la Souris, GervaIs (1972) n'a observé aucune immunofluorescence des spermatozoïdes dans des coupes de tissu testiculaire. 
Cet auteur estime que les procédés expérimentaux qu'il utilise sont insuffisamment sensibles compte tenu de la faible concentration de ces antigènes sur les spermatozoïdes.

Cependant, Erickson (1972 $a$ et $1972 b$ ) a décrit deux techniques élégantes pour la détection d'antigènes $\mathrm{H}-2$ sur des spermatozoïdes de Souris : l'immunofluorescence sur des spermatozoïdes vivants et la liaison d'anticorps marqués avec ${ }^{125} \mathrm{I}$ dans la technique d'antiglobuline. Cependant ERICKson a notamment appliqué ces techniques à la détection d'antigènes comportant la spécificité 5 , très soluble et, comme il le reconnaît lui-même, ses résultats ne permettent pas de manière générale, de trancher entre l'hypothèse d'une expression directe des antigènes du système $\mathrm{H}-2$ sur les spermatozoïdes, et celle de leur adsorption passive. Nous pensons que les résultats d'ERICKSON ne fournissent aucune preuve contredisant l'hypothèse d'expression d'une partie de génome sur la surface du spermatozoïde.

PADma (1972) a noté l'absence d'antigènes communs entre érythrocytes et spermatozoïdes chez le Lapin, mais ceci en n'utilisant que des antisérums anti -A, -D et -F. Comme les spermatozoïdes n'avaient pas été lavés avant addition de la suspension indicatrice dans la modification de la technique d'agglutination de cellules mixtes qu'utilisait cet auteur, ses expériences ont perdu en précision et en sensibilité ce qu'elles gagnaient en simplicité. Ces résultats négatifs ne prouvent donc pas l'absence de ces antigènes du système $\mathrm{Hg}$ sur les spermatozoïdes du Lapin.

\section{C. - DISCUSSION ET CONCLUSION}

Cette étude bibliographique met bien en évidence la grande diversité des techniques qui ont été mises en œuvre par les équipes qui se sont intéressées à l'immunologie des spermatozoïdes. Il semble que dans l'ensemble, les études concluant à l'existence d'antigènes sur les spermatozoïdes soient plus convaincantes que celles qui rapportent un résultat négatif. En fait, dans ces dernières, la plupart des auteurs n'ont pas tenu compte de certains facteurs dans le choix des techniques immunologiques à utiliser :

I ${ }^{0}$ La quantité d'alloantigènes présents sur les spermatozoïdes est faible. VoJTiskova et Pokorna (1972) ont estimé la quantité des antigènes $\mathrm{H}-2$ présents sur les spermatozoïdes de Souris au huitième de celle trouvée sur les cellules de la rate et ERICKSON (I972 a) au dixième de celle trouvée sur les lymphocytes. De plus, la surface de la membrane des spermatozoïdes est beaucoup plus faible que celle de ces cellules.

$2^{\circ} \mathrm{A}$ l'exemple des antigènes du groupe sanguin humain $\mathrm{A}$ que l'on trouve dans le plasma séminal, certains alloantigènes sont présents dans les sécrétions. Les études qui concluent à la présence de ces antigènes sur les spermatozoïdes n'apportent donc pas une preuve définitive de l'expression du génome des spermatozoïdes. L'analyse devrait porter sur un nombre significatif de donneurs non sécréteurs.

$3^{\circ}$ La spécificité de l'agglutination des spermatozoïdes dans les techniques usuelles d'agglutination simple ou mixte n'est pas étroite. Ces techniques immunologiques devraient donc être modifiées pour leur application à des travaux sur les spermatozoïdes.

$4^{\circ}$ Les cellules autres que les spermatozoïdes et les débris cellulaires présents dans les éjaculats interfèrent dans les tests immunologiques.

$5^{\circ}$ Dans les tests de cytotoxicité par exemple, les conditions physicochimiques auxquelles sont soumis les spermatozoïdes doivent être particulièrement douces pour préserver leur viabilité.

Il est certain que les résultats contradictoires publiés par les diverses équipes, aussi bien que les conclusions opposées qu'elles en tirent, compliquent considérablement l'approche de ce domaine de recherche. Il semble, cependant, que des résultats expérimentaux suffisants ont été accumulés, à l'aide de techniques immunologiques, pour conclure à la présence d'antigènes d'au moins un système d'histocompatibilité principal sur les spermatozoïdes de l'Homme et de la Souris. Il 
reste cependant à établir si les antigènes présents sont commandés par le génome diploïde de l'individu ou, au contraire, par la fraction haploïde du spermatozoïde.

L'interprétation statistique d'un test de cytotoxicité soigneusement conçu pourrait fournir une réponse à cette question. Malheureusement, les données disponibles ne sont pas concluantes. En effet, dans les travaux de Fellous et Dausset (1970) et de Goldberg et al. (1970 et r971) des anticorps cytotoxiques purifiés auraient dû être utilisés pour obtenir une destruction presque totale des spermatozoïdes. Par ailleurs, les témoins auraient pu donner des niveaux de cytotoxicité plus faibles si l'on avait séparé les spermatozoïdes morts des spermatozoïdes mobiles avant les essais de cytotoxicité à l'aide de gradients de densité semblables à ceux utilisés pour les spermatozoïdes bovins (PRAKASH, 1973) et en employant des tampons plus appropriés.

L'index de spermatotoxicité mis au point par HAMMERLyNCK et Rumke (I968) permet d'aboutir à des conclusions plus significatives. L'application de cet index aux données de FELLous et DAUSSET (1970) conduit à des résultats qui tendent à confirmer l'hypothèse selon laquelle les antigènes décelés par ces auteurs seraient déterminés par une fraction haplö̈de du génome.

Quelques auteurs ont suggéré l'existence, sur les spermatozoïdes, de membranes mixtes, d'origine partiellement diploïde et partiellement haploïde. Cette hypothèse n'a été confirmée par aucune preuve expérimentale : il reste, en effet, à démontrer que des fragments de membranes d'origine diploïde sont présents tout au long des stades de maturation du spermatozoïde, donc pendant plusieurs semaines. Au contraire, Loor et al. (1972) ont récemment montré un phénomène de resynthèse des récepteurs de surface de lymphocytes qui s'opère en $4^{-5}$ heures au cours de la phase de repos.

Parmi les auteurs qui ont envisagé la possibilité que le génome haploïde du spermatozoïde, puisse faire preuve d'une certaine activité, BEATTY (1972) a discuté cette hypothèse en se basant sur les études de FAwCETT (1972) au microscope électronique. La fermeture passagère des ponts entre les spermatocytes secondaires pourrait permettre l'expression des gènes haploïdes sur un court intervalle de temps. Récemment, dans le laboratoire de Vorsin (1973), la présence d'autoantigènes sur les spermatozö̈des du Cobaye a été établie au cours de la spermatogenèse et seulement après la fin de la méiose.

Des études récentes ont montré qu'il y a transcription et traduction du seul ADN mitochondrial haploïde des spermatozoïdes bovins éjaculés (Premkumar et BHARgava, I972). Dans ces cellules, l'ADN nucléaire reste inerte. Les résultats négatifs antérieurs portant sur le métabolisme d'acides nucléiques des spermatozoïdes pourraient s'expliquer par l'intervention du plasma séminal dans les études in vitro. On sait, en effet, que le plasma séminal contient un inhibiteur de la synthèse de l'ARN et de la ribonucléase active.

Sheid et Wilson (1970 $a$, 1970 $b$, 197 $a$, 197 $b$ ) ont démontré que la méthylation de l'ARN subissait une puissante inhibition due à la dégradation par le plasma séminal du substrat des $t$-RNA en polynucléotides. Ces mêmes auteurs ont démontré l'hydrolyse de toutes les espèces d'ARN en présence du plasma séminal bovin.

Si certains antigènes de la membrane des spermatozoïdes sont déterminés par un génome haploïde, la suggestion suivante faite par LiNDSLEY (1972) semble particulièrement séduisante :

" ... it seems to me that in all these cases the phenotypes of sperm (either with respect to trypan blue uptake or agglutinability) is being determined and sperm genotype inferred, why is it not possible after antibody (specific) treatment of sperm from heterozygous male to test the genotype of surviving sperm by using them in artificial insemination and determining the genotype of the resulting progeny."

Cette expérience est certainement envisageable, sa méthodologie ne posant pas de problèmes insurmontables. C'est ainsi, par exemple, que des lymphocytes $T$ qui contiennent le marqueur $\theta$ peuvent être détruits spécifiquement par des anticorps anti- $\theta$ d'une population de lymphocytes $\mathrm{T}$ et $\mathrm{B}$. Des expériences rigoureusement conçues devraient pouvoir être entreprises dans un avenir raisonnable. 


\title{
REMERCIEMENTS
}

Je remercie F. GrosclaUde pour sa participation à l'élaboration et à la mise au point dece texte, ainsi que MM. J. Martinet, P. Millot et Mlles Christine Lorsignol et Odile About pour leur aide dans la traduction du manuscrit anglais.

\section{SUMMMARY}

\author{
THE ALLOANTIGENS ON MAMMALIAN SPERMATOZOA \\ THEIR CONTROL BY THE HAPLOÏD GENOME
}

This review summarises the attempts made to detect the alloantigens on mammalian spermatozoa. These published reports concern the antigens of $\mathrm{ABO}, \mathrm{MNS}, \mathrm{Rh}, \mathrm{P}\left(\mathrm{Tj}^{a}\right), \mathrm{Xg}^{a}$ and HL-A systems of man, A, B, C, F, Z, M and J systems of cattle) $\mathrm{H}-2$ and $\mathrm{H}-\mathrm{Y}$ systems of mice and $\mathrm{Hg}$ system of rabbits.

The discordance in the published results and conclusions is largely due to the use of faulty technics. However, in the light of the present understanding of the immunological concepts, it appears that studies demonstrating the presence of certain alloantigens on spermatozoa are more convincing than those indicating their absence.

Though it has not yet been clearly demonstrated that the synthesis of these antigens is controlled genetically by the haploid genome of the carrier cells, recent work lends indirect support to this possibility. Moreover this seems compatible with our present knowledge of the biology of spermatozoon.

The separation of individual spermatozoal population by use of immunological techniques could thus be envisaged in the near future.

\section{RÉFÉRENCES BIBLIOGRAPHIQUES}

Ackerman D. R., 1969. The adsorption of anti-A and anti-B antibodies on human spermatozoa. Fert. Steril, 20, 324-333.

Barth R. F., Russell P. S., r964. The antigenic specificity of spermatozoa I. An immunofluorescent study of the histocompatibility antigens on mouse sperm. J. Immunol., 93, 13-19.

Beatty R. A., 1970. The genetics of the mammalian gamete. Biol. Rev., 45, 73-Irg.

Beatty R. A., I972. Dans la discussion de FAwCett D. W., I972. Observations on cell differentiation and organelle continuity in spermatogenesis in Beatty R. A., and Gluecksohn-Waelsch S., Proc. Int. Symp. The Genetics of the spermatozoon. Univ. Edinburgh. 37-68.

Boettcher B., I965. Human ABO blood group antigens on spermatozoa from secretors and nonsecretors. J. Reprod. Fertil., 8, 267-268.

Сонen J., r97r. The comparative physiology of gamete population. Advances Comp. Physiol. and Biochem., 4, 268-380.

Coombs R. R. A., Bedford D., Roulllard L. M., 1956. A and B blood group antigens on human epidermal cells. Lancet, 1, 46I-463.

Docton F. L., Ferguson L. C., Lazear E. J., Ely F., 1952. The antigenicity of bovine spermatozoa. J. Dairy Sci., 35, 706-709.

Edwards R. G., Ferguson L. C., Coombs R. R. A., r964. Blood group antigens on human spermatozoa. J. Reprod. Fert., 7, 153-r6r.

Erickson R. P., I972 a. Alternative modes of detection of $\mathrm{H}-2$ antigens on mouse spermatozoa. In Proc. Int. Symp. The Genetics of the Spermatozoon, ed. Beatty R. A. and Gluecksohn-WaElsch, S. Univ. Edinburgh, I9I-202.

ERICKson R. P., r $1972 b$. An antiglobulin test for the detection of surface antigens of mouse spermatozoa. J. Reprod. Fert.; 28, ro9-III. 
FawcetT D. W., r972. Observations on cell differentiation and organelle continuity in spermatogenesis. In Proc. Int. Symp. The Genetics of the Spermatozoon, ed. Beatty R. A. and Gluecksohn-Waelsch S., Univ. Edinburgh, 37-68.

Fellous M., I969. Thèse, Faculté de Médecine, Université de Paris.

Fellous M., DaUSSet J., I97o. Probable haploid expression of HL-A antigens on human spermatozoon. Nature, London, 225, I9I-I93.

Friedenreich V., Hartmann G., I938. Ueber die Verteilung der Gruppenantigene in Organismus der sogenannten "Ausscheider " und "Nichtausscheider". Z. Immun. Forsch., 92, I4I-I5I. Cité par RAcE R. R., SANGER F., I962.

Gervais A. G., I972. Failure to detect H-2 antigens in mouse testis and heart by immunofluorescence. Folia Biol., 18, 50-52.

Goldberg E. H., Aoki T., Boyse E. A., Bennetr D., r97o. Detection of H-2 antigens on mouse spermatozoa by the cytotoxicity test. Nature, 228, 570-572.

Goldberg E. H., Boyse E. A., Bennett D., Scheid M., CArswell E. A., I97I. Serological demonstration of H-Y (male) antigens on mouse sperm. Nature, 232, 478-480.

Goldberg E. H., I972. Ph. D. thesis. Cornell University Dissertation Abs. Int. Series B, 32 Part I $6850-\mathrm{B}-685 \mathrm{I}-\mathrm{B}$.

Gullbring B., I957. Investigation on the occurrence of blood group antigens from man and serological demonstration of the segregation of characters. Acta. Medica. Scand., 159, r69-172.

Hamerlynck J., Rumke P., I968. A test for the detection of cytotoxic antibodies to spermatozoa in man. J. Reprod. Fert., 17, I9I-194.

HartmanN G., I94I. Group antigens in human organs. Munksgaard, Copenhagen. Cité par RACE R. R., SANGER F., I962.

Holborow E. J., Brown P. C., Glynn L. E., Hawes M. D., Gresham G. A., O'Brien T. F., Coombs R. R. A., I96o. The distribution of the blood groupe Á antigen in human tissues. Brit. $\dot{J}$. Exp. Path., 41, 430-437.

Krainskaya-Ignatova V. N., r929. Blood group properties of sperm. Vrachebnoe delo, 8, 533 (en russe). Z. gerichtl. Med., 13, 44I. Cité par Vulchanov V. H., Popivanov R., I972.

LANDSteiner K., Levine P., I926. On group specific substances in human spermatozoa. J. Immunol., 12, 4I5-4I8.

LEHRS H., I930. Ueber gruppenspezifische Eigenschaften des menchlichen Speichels. Z. Immunol. Forsch., 66, I75-I92. Cité par RACE R. R., SANGER F., I962.

Levine P., Celano M. J., I96r. The question of $\mathrm{D}\left(\mathrm{Rh}_{\mathbf{0}}\right)$ antigenic sites on human spermatozoa. Vox sang., 6, 720-723.

LinDSLEY, I972. Dans la discussion de ERICKSON R. P., I972. Alternative modes of detection of H-2 antigens on mouse spermatozoa. In. Proc. Int. Symp. The Genetics of the spermatozoa ed. BeAtty R. A., GLueckSOHN-WAELSCH S., Univ. Edinburgh, I9I-202.

Loor F., Forni L., Pernis B., I972. The dynamic state of the lymphocyte membrane factors affecting the distribution and turnover of surface immunoglobulins. Eur. J. Immunol., 2, 203-2r2.

Maligina A. A., I949. Testing the group specificity of semen in forensic medical practise. Trudi Gnii Inst., Sudebn. Med., p. I69 (en russe). Cité par Vulchanov V. H., Popivanov R., I972.

Metchnikoff E., I899. Études sur la résorption des cellules. Ann. Inst. Pasteur, 13, 737-77o.

Mетснлікоғғ E., rgoo. Recherches sur la spermatotoxine et l'antispermatoxine. Ann. Inst. Pasteur, 14, I-I3.

METZ C., I967. Gamete surface components and their role in fertilization. Fertilization ed. METz C., Monroy A., Academic Press. New York, 1, r63-236.

Morgenstern L. L., I968. ABO blood group antigens on human spermatozoa. VIth Int. Cong. Physiol. Reprod. A. I., Paris, Vol. I, 557-559.

PADMA M. C., I969. Relationship of blood group antigens in cattle to homologous spermatozoan antigens as detected by natural agglutinins. Ind. J. Exp. Biol., 7, 246-249.

PADMA M. C., I.972. Relationship of isoantigens of rabbit spermatozoa to erythrocyte antigens. J. Reprod. Fert., 31, I-6.

Papivanov R., Ermenkova L., I960. Studies on group properties of human spermatozoa by means of absorption and elution. Izvest. Inst. Fisiol. Ban, 4, 441-445 (en bulgare).

Papivanov R., Vulchanov V. H., I962. Segregation of man's AB group spermatozoa in A- and Bspermatozoa through agglutination with immune anti-A rabbit serum. C. R. Acad. Sci. Bulg., 15, $669-672$.

Papivanov R., Vulchanov V. H., I969. Immunogenetics of human spermatozoa in Immunology and Reproduction ed. EDwards R. G. International Planned Parenthood Federation, London, I55-I67.

Parish W. E., Carron-Brown J. A., Richards C. B., ig67. The detection of antibodies to spermatozoa and to blood group antigens in cervical mucus. J. Reprod. Fert., 13, 469-483.

Ponder E., 1948. Hemolysis and related phenomenon. Grune and Stratton New-York.

PraKash C., I973a. Séparation des spermatozoïdes mobiles des autres cellules et des débris cellulaires présents dans le sperme bovin. Ann. Biol. anim. Biochim. Biophys., (sous presse). 
PRAKash C., r973 $b$. Manuscrit en préparation.

Premkumar E., Bhargava P.M., I972. Transcription and translation in bovine Spermatozoa. Nature, (New Biol.), 240, I39-143.

Putkonen T., r930. Ueber die gruppenspezifischen Eigenschaften verschiedener Körperflüssigkeiten. Acta. Soc. Med. Fenn. Duodecim. A., 14, ro7. Cité par Race R. R., Sanger R., ig62.

Quinlivan W. L. G., Masouredis S. P., I962. $\mathrm{Rh}_{0}(\mathrm{D})$ antigen content of human spermatozoa. Immunology, 5, 267-277.

Race R. R., Sanger R., I962. Blood Groups in Man. Blackwell Scientific Publications, Oxford, 4th edition.

Schmid D. O., Conneally P. M., Stone W. H., I964. Blood Group antigens on bull spermatozoa. J. Anim. Sci., 23, I I98-rig9 (Abs.).

Schultz-LARSEN J., I958. The morphology of the human sperm : electron microscopic investigations of the ultrastructure. Acta. Pathol. Microbiol. Scand. Suppl., I28, I-I2r.

Shahani S., Southam A., I962. Immunofluorescent study of the ABO blood group antigens in human spermatozoa. Am. J. Obstet. Gynec., 84, 660-666.

Sheid B., Wilson S. M., I970 a. Inhibition of transfer RNA methylases by bull semen. I. Localisation, identification and properties of the inhibitor. Biochem. Biophys. Acta, 224, 382-39o.

Sheid B., Wilson S. M., I97o $b$. t-RNA methylase inhibitor(s) in bull sperm. 54th Ann. of the Federation of American Societies for experimental biology., Atlantic City, April 12-17 Fed. Proc., 29, 3883 (Abstract).

Sheid B., Wilson S. M., I97 I $a$. Necessity for restudying nucleic acid and protein synthesis in mammalian semen. Nature, 232, 628-629.

Sheid B., Wilson S. M., I97I $b$. Non-enzymatic hydrolysis of transfer RNA by bull semen. Life Sci., Part. II, 9I9-926.

Shulman S., I97I $a$. Immunity and infertility. Contraception, 4, I35-154.

Shulman S., I97 $b$. Antigenicity and autoimmunity in sexual reproduction : A review. Clin. Exp. Immunol., 9, 267-288.

Shulman S., I972. Immunologic barriers to fertility. Obstet. Gynecol. Survey, 27, 553-606.

Sjovall H., r948-I950. An attempt to differentiate between homozygotes and heterozygotes of the ABO system by examining the spermatozoa. Acta Genet. Statist. Med., 1, 245-25I.

Spooner R. L., 1972. Dans la discussion de Vulchanov V. H., Popivanov R., 1972. Immunological data suggesting post-segregational gene action in human spermatozoa in Proc. Int. Symp. The Genetics of the spermatozoa, ed. Beatty R. A., Gluecks-Waelsch S., Univ. Edinburgh.

Stone W. H., IRwin M. R., r954. The J substance of cattle : x. Development and immunogenetic studies. J. Immunol., 73, 397-406.

Stormont C., I949. The acquisition of J substance by bovine erythrocytes. Proc. Natl. Acad. Sci., 35, 232-237.

Tyler A., r96r. Approaches to the control of fertility based on immunological phenomenon. $J$. Reprod. Fertil., 2, 473-506.

Voisin G. A., 1973. (Communication personnelle).

Vojtiskova M., Polackova M., РокоRna Z., ig69. Histocompatibility antigens on mouse spermatozoa. Folia. Biol., 15, 322-332.

Vojtiskova M., Pokorna Z., I972a. Cellular antigens of mouse spermatozoa as possible markers of gene action. Proc. Int. Symp. Tne Genetics of the Spermatozoan. ed. Beatty R. A., GluecksohnWaElsch S., Univ. Edinburg, r6o-176.

Vojtiskova M., Pokorna Z, I972 b. Developmental expression of $\mathrm{H}-2$ antigens in the spermatogenic cell series : Possible bearing on haploid gene action. Folia Biol., 18, I-9.

Vulchanov V. H., Papivanov R., I972. Immunological data suggesting post segregational gene action in human spermatozoa. Proc. Int. Symp. The Genetics oft he Spermatozoan. ed. BEATty R. A., Gluecks-Waelsch S., Univ. Edinburgh, r77-I9o.

YAMAKAMI K., 1926. The individuality of semen with reference to its property of inhibiting specifically iso-hemoagglutination. J. Immunol., 12, I85-I89.

Ycas M. K. M., I949. Studies on the development of a normal antibody and of cellular antigens in the blood of sheep. J. Immunol., 61, 327-347. 